\title{
Comparison of Percutaneous Nephrolithotomy and Ureteroscopic Lithotripsy for Upper Ureteral Calculi
}

\author{
Jiyu Zhao ${ }^{1,2, *, \dagger}$, Sheng Yang ${ }^{1,2, \dagger}$, Zejun Xiao ${ }^{3, \dagger}$, Jun Zhang ${ }^{1,2}$, Jia Liu ${ }^{1,2}$, Ying Qi1 ${ }^{1,2}$, \\ Xianen $\mathrm{Gu}^{1,2, *}$
}

${ }^{1}$ Department of Urology, Beijing ChuiYangLiu Hospital, Beijing, P. R. China ${ }^{2}$ Department of Urology, ChuiYangLiu Hospital affiliated to Tsinghua University, Beijing, P. R. China

${ }^{3}$ Department of Urology, Cancer Hospital Chinese Academy of Medical Science, Beijing, P. R. China

*Correspondence
yu_19821@163.com
(Jiyu Zhao)
guxen@sina.com
(Xianen Gu)
† These authors contributed equally.

\begin{abstract}
This study aimed to investigate the efficacy, safety and cost of ureteroscopic lithotripsy (URSL) and percutaneous nephrolithotomy (PCNL) for large proximal ureteral calculi. We retrospectively reviewed the outcomes of 205 subjects with ureteral stones size $\geq 1$ $\mathrm{cm}$ from January 2011 to December 2017. We divided URSL into two groups including URSL + S with Stone Cone and URSL without Stone Cone. Ninety subjects were treated with URSL, fifty subjects were treated with URSL $+\mathrm{S}$ and sixty-five subjects were treated with PCNL. The success rate was $73.3 \%, 96.0 \%$ and $96.6 \%$ in URSL, URSL $+\mathrm{S}$ and PCNL groups, respectively. The treatment time in PCNL group $(77.3 \pm 19.1$ minutes) was longer than URSL group ( $42.0 \pm 16.6$ minutes) and URSL+S group (55.6 \pm 20.3 minutes), and the hospital stay was longer in PCNL ( $9.5 \pm 1.6$ days) group than in URSL group $(2.4 \pm 4.8$ days $)$ and URSL $+\mathrm{S}$ group $(2.0 \pm 2.4$ days $)$. The cost-effectiveness ratio was 124.6, 159.1 and 135.3 in URSL, URSL + S and PCNL groups, respectively. In conclusion, Stone Cone could increase treatment success rate but incur higher cost. URSL is considered as the best cost-effective option, and the patients without medical insurance tend to choose PCNL because of high success rate of treatment.
\end{abstract}

\section{Keywords}

Lithotripsy, Ureteral calculi, Cost-benefit analysis

\section{Introduction}

Large proximal ureteral stones are usually followed with urinary obstruction, which may lead to renal insufficiency and urosepsis. However, the most appropriate option for treating those stones is still controversial [1]. Extracorporeal shock wave lithotripsy (SWL), ureteroscopic lithotripsy (URSL) and percutaneous nephrolithotomy (PCNL) are used for the removal of ureteral stones in proximal ureteral calculus according to the European Association of Urology (EAU) Guideline [2]. However, SWL is not a best choice, especially for large, impacted proximal ureteral calculus due to poor overall success rates. URSL is considered as a minimally-invasive treatment and PCNL dramatically increased stone-free rate for upper ureteral stones [3]. However, each treatment has limitations. Migration of stones is a common phenomenon in URSL and several devices have been applied to prevent stone migration such as Stone Cone which increases stone-free rate but also increases the cost [4]. On the other hand, PCNL has increased transfusion rate because it is an invasive technique [5]. Therefore, it is a challenge to choose treatment for the patients with large proximal ureteral stones. In this retrospective study, we aimed to investigate the clinical effectiveness, safety and costeffectiveness of URSL and PCNL for large proximal ureteral calculus.

\section{Subjects and Methods}

\subsection{Subjects}

All subjects with proximal ureteral calculus (with stone of diameter $\geq 1.0 \mathrm{~cm}$ in proximal ureter) diagnosed by computed tomography (CT) imaging between January 2011 and December 2017 at Chuiyangliu Hospital were included. Patients with kidney stone and anomalies of the urinary tract (e.g. duplex system, horseshoe kidney, previous ureteral surgery history) were excluded.

\subsection{Operations}

All subjects were divided to two groups to receive treatment with URSL or PCNL based on their preference. URSL was performed following standard procedure as follows. The ureteral calculi was approached using a rigid ureteroscope (Wolf 8/9.8 F, Richard Wolf, Germany) followed by a guide wire. To prevent the calculi from drifting to the pelvis, basket devices were used selectively. Furthermore, URSL was subdivided into two subgroups including URSL+S with the use of Stone Cone (Boston Scientific, Natick, USA) and URSL without the use of Stone Cone. PCNL was performed 
TA B L E 1. Demographic and clinical characteristics of the study population.

\begin{tabular}{|c|c|c|c|c|}
\hline Variable & & URSL $(n=140)$ & PCNL $(n=65)$ & $\mathbf{p}$ \\
\hline \multirow[t]{2}{*}{ Gender } & Male & $107(76.4 \%)$ & $48(73.8 \%)$ & \multirow[t]{2}{*}{0.823} \\
\hline & Female & $33(23.6 \%)$ & $17(26.2 \%)$ & \\
\hline Age (year) & & $52.3(11.2)$ & $50.8(13.3)$ & 0.556 \\
\hline \multirow[t]{2}{*}{ Stone side } & Left & $90(64.3 \%)$ & $37(56.9 \%)$ & \multirow[t]{2}{*}{0.393} \\
\hline & Right & $50(35.7 \%)$ & $28(43.1 \%)$ & \\
\hline Stone size $(\mathrm{cm})$ & & $1.7(0.2)$ & $1.8(0.6)$ & 0.083 \\
\hline \multirow[t]{3}{*}{ Comorbidities } & Diabetes mellitus & 7 & 5 & 0.663 \\
\hline & Ischemic heart disease & 6 & 4 & 0.823 \\
\hline & hypertension & 11 & 7 & 0.671 \\
\hline \multirow[t]{2}{*}{ Insurance status } & Insurance & 130 & 47 & \multirow[t]{2}{*}{0.004} \\
\hline & Without insurance & 10 & 18 & \\
\hline
\end{tabular}

following standard procedure as follows. The subjects were positioned in lateral position and a pigtail catheter was placed into the collecting system under cystoscopy, and then an $18 \mathrm{G}$ access needle was introduced to the targeted calyx guided by ultrasonography. Holmium YAG Laser (Karl Storz) with $550 \mu \mathrm{m}$ fiber probe (pulse frequency: $8-10 \mathrm{~Hz}$, power supply: $9.6-16 \mathrm{~W}$ ) were used for intracorporeal pneumatic lithotripsy to fragment the stone. The subjects received general or combined spinal and epidural anesthesia in URSL and PCNL group.

\subsection{Measurements}

Included subjects had data available on demographic characteristic, stone diameter, medical expenses and follow-up information. The following situations were defined as failure: stone drifted into the pelvicalyceal system or the endoscope cannot approach the stone for any reason (e.g. ureterostenosis, ureteral polyps) in URS group. Kidney, ureter, bladder (KUB) imaging was performed before discharge from hospital and CT imaging was used to evaluate the efficacy one month after the last treatment. Stone-free confirmed by CT imaging (clinically insignificant residual fragment $<3 \mathrm{~mm}$ ) and no symptom were defined as a successful treatment, and was regarded as the criteria for discharge from hospital. Operation-related complications measured by Clavien-Dindo classification were recorded, including hematuria, fever, ureteral perforation and transfusion. Postoperative stenting and postoperative protocol for follow-up followed the European Association of Urology (EAU) Guideline [2].

\subsection{Statistical analysis}

Statistical analysis was performed using SPSS version 24.0. Categorical and continuous variables were detected with twotailed Chi-square tests and a two-tailed Student's t tests, respectively. Multiple groups were compared with one-way ANOVA. P-value less than 0.05 was considered as significant.

\section{Results}

\subsection{Characteristics of the patients}

The general characteristics of the subjects were listed in Table 1 . One hundred and forty patients were treated with URSL and sixty-five patients were treated with PCNL. The characteristics of both groups showed no significant differences in the gender, age, stone side and mean size of stone $(\mathrm{p}>$ $0.05)$. However, the preference of patients with and without medical insurance was different. The patients without medical insurance preferred PCNL (Table 1).

\subsection{Treatment outcome}

The success rate after the first treatment was $73.3 \%, 96.0 \%$ and $96.9 \%$ in URSL, URSL + S and PCNL groups, respectively. The success rate of URSL with Stone Cone was higher than that without the device and the difference was statistically significant. As expected, PCNL group had longer duration of operation (77.3 minutes) and hospital stay (9.5 days) than the other two groups. However, there were no significant differences in ureteral stricture and polypus in three groups (Table 2).

\subsection{Operation-related complications}

Patients who underwent PCNL were associated with a higher risk of hematuria (29.2\%) compared to other two groups $(5.6 \%$ in URSL and $8.0 \%$ in URSL $+\mathrm{S}$ ). There was no significant difference in fever among the groups $(p=0.748)$. There were two cases of ureteral perforation during URSL, and one patient need transfusion (Table 3).

\subsection{Cost profile}

PCNL group had a longer hospital stay and a higher cost than the other groups except medical instrument $(p<0.05)$. The cost increased in URSL $+\mathrm{S}$ group because of expensive cost 
TA B L E 2. Comparison of treatment outcome.

\begin{tabular}{|lcccc} 
& URSL $(\mathbf{n}=\mathbf{9 0})$ & URSL + S (n= 50) & PCNL (n= 65) & p \\
\hline Treatment success after first treatment & $66(73.3 \%)$ & $48(96.0 \%)$ & $63(96.9 \%)$ & $<0.0001$ \\
Mean (SD) overall treatment time (min) & $42.0(16.6)$ & $55.6(20.3)$ & $77.3(19.1)$ & $<0.0001$ \\
Mean (SD) hospital stay (day) & $2.4(0.8)$ & $2.0(0.4)$ & $9.5(1.6)$ & $<0.0001$ \\
Ureteral stricture/polypus & $69(76.7 \%)$ & $42(84.0 \%)$ & $53(81.5 \%)$ & 0.543 \\
\hline
\end{tabular}

Continuous variables were presented as the mean (standard deviation), and categorical variables were expressed as number (proportion). P for trend was detected with one-way ANOVA and Fisher exact probability test. URSL, ureteroscopic lithotripsy. URS $+S$, ureteroscopic lithotripsy with Stone Cone. PCNL, percutaneous nephrolithotomy.

TA B L E 3. Comparison of operation-related complications.

\begin{tabular}{lcccc|} 
Operation-related complications & URSL(n= 90) & URSL + S $(\mathbf{n = 5 0 )}$ & PCNL $(\mathbf{n = 6 5})$ & $\mathbf{p}$ \\
\hline Hematuria (Clavien I), n (\%) & $5(5.6)$ & $4(8.0)$ & $19(29.2)$ & $<0.001$ \\
\hline Fever (Clavien II), n (\%) & $2(2.2)$ & $2(4.0)$ & $3(4.6)$ & 0.748 \\
\hline Ureteral perforation (Clavien II), n (\%) & $2(2.2)$ & 0 & 0 & - \\
Transfusion, n (\%) & 0 & 0 & $1(1.5)$ & - \\
\hline Total, n (\%) & $9(10)$ & $6(12)$ & $23(35.3)$ & $<0.001$ \\
\hline
\end{tabular}

p for trend was detected with Chi-Square. URSL, ureteroscopic lithotripsy. URS $+S$, ureteroscopic lithotripsy with Stone Cone. PCNL, percutaneous nephrolithotomy.

of medical instrument ( $¥ 8522.4$ ), which made up $55.8 \%$ of total cost (¥15273.6) (Table 4).

\subsection{Cost-effectiveness ratio analyses}

The surgical success rate increased from $73.8 \%$ to $96.0 \%$ after the use of Stone Cone, but cost-effectiveness ratio in URSL+S group was higher than that of the other groups. Although URSL group had the lowest success rate, its cost-effectiveness ratio (124.6) was the best in three groups (Table 5).

\section{Discussion}

With the development of techniques, more options for the treatment of proximal ureteral calculus are available, ranging from open to minimal invasive surgery such as laparoscopic ureterolithotomy, PCNL, URSL, flexible ureteroscopy and lasertripsy (fURSL) and ESWL. Open and laparoscopy surgery are not first-line treatment unless other options fail or adjacent organs need to be interfered at one-stage operation [3]. As a well-tolerated procedure, SWL is the primary treatment option for proximal ureteral calculus, but it has a poor overall success rate for large, impacted proximal ureteral calculus $[6,7]$. The fURS is a new operation procedure for urinary calculus [8], but we only began the technique since 2016 and have very limited follow-up information. Therefore, in this study we only compared the subjects treated with URSL and PCNL.

URSL has been regarded as an efficacious technique in the treatment of ureteral stones, but some challenges limit its use [9]. One cause of failure during URSL is stone migration (retropulsion) into the kidney [4]. The retropulsion rate ranged from $2.0 \%$ to $15.5 \%$ in some studies $[10,11]$. Therefore, several devices were applied to prevent stone migration during ureteric lithotripsy $[12,13]$. In present study, we used Stone Cone, and treatment success rate $(96.0 \%)$ with the use of
Stone Cone was higher than that $(73.3 \%)$ without the use of this device. Similar study reported that the migration rate was significantly lower in the group using Stone Cone (4.5\%) compared to control group (31.8\%) [12]. We also paid attention to ureteral perforation which is the most common and serious complications in URSL. The reported perforation rate ranged from $3.8 \%$ to $4.9 \%[14,15]$. In this study, only two patients had ureteral perforation and it was managed by double J stent.

Although the percutaneous approach became more advanced such as mini-, ultramini- and micro PCNL, it may cause complications such as transfusion, adjacent organ injury, sepsis [16]. Our finding in this study is consistent with that bleeding is common during PCNL which has higher hematuria and transfusion rate. A similar study compared percutaneous antegrade and retrograde approach for impacted upper-ureteral stone and showed that PCNL was an effective option, especially in the cases with moderate to severe hydronephrosis [17]. Another study showed that the stone-free rate was $95.4 \%$ in the PCNL group and 58\% in the URSL group [18]. In this study, we achieved a stone-free rate of $96.6 \%$ in PCNL group, which was higher compared to two other groups. Therefore, we postulate that PCNL is an effective procedure for impacted upper-ureteral stone, because of higher stone clearance rate.

On the other hand, cost escalating of health expenditure is a global problem [19, 20]. Increasing national wealth alone does not necessarily increase national health [21, 22]. Urinary lithiasis is one of the most common afflictions of modern society [23]. Given the high frequency with which stones occur and recur, the development of appropriate treatment program is desirable [3]. Ursiny et al. reported the cost effectiveness of anti-retropulsion devices for ureteroscopic lithotripsy and the average cost of the device was $\$ 278$. Considering the estimated 
TA B L E 4. Comparison of cost.

\begin{tabular}{lcccc} 
Cost & URSL $(\mathbf{n}=\mathbf{9 0})$ & URSL + S $(\mathbf{n}=\mathbf{5 0})$ & PCNL $(\mathbf{n}=\mathbf{6 5})$ & $\mathbf{p}$ \\
\hline Medicine $(¥)$ & $2036.2(824.8)$ & $2279.6(783.1)$ & $2648.3(1020.7)$ & 0.020 \\
\hline Image examination $(¥)$ & $1187.4(565.9)$ & $1262.12(523.5)$ & $1590.3(432.0)$ & 0.006 \\
\hline Anesthesia ( $¥)$ & $191.1(28.5)$ & $187.0(4.1)$ & $202.7(26.2)$ & 0.044 \\
\hline Operation $(¥)$ & $2753.0(704.2)$ & $2529.2(616.1)$ & $2988.0(354.3)$ & 0.023 \\
\hline Medical instrument $(¥)$ & $2411.4(1477.3)$ & $8522.4(2773.4)$ & $4793.5(893.1)$ & 0.006 \\
\hline Nursing $(¥)$ & $551.6(485.5)$ & $493.3(269.8)$ & $845.6(646.0)$ & 0.020 \\
\hline Total $(¥)$ & $9130.7(2530.3)$ & $15273.6(3170.9)$ & $13068.3(1918.2)$ & $<0.0001$ \\
\hline
\end{tabular}

The costs were continuous variables that presented as the mean (standard deviation). P for trend was detected with one-way ANOVA. URSL, ureteroscopic lithotripsy. URS $+S$, ureteroscopic lithotripsy with Stone Cone. $P C N L$, percutaneous nephrolithotomy.

TA B L E 5. Cost-effectiveness ratio (CER) for different strategies of proximal ureteric stone.

\begin{tabular}{lccc} 
& Mean hospitalization costs $¥)$ & Success rate $(\%)$ & Cost-effectiveness ratio \\
\hline URSL $(\mathrm{n}=90)$ & 9130.7 & 73.3 & 124.6 \\
\hline URSL + S $(\mathrm{n}=50)$ & 15273.6 & 96.0 & 159.1 \\
\hline PCNL $(\mathrm{n}=65)$ & 13068.3 & 96.6 & 135.3 \\
\hline
\end{tabular}

Cost-effectiveness ratio $=$ Mean hospitalization costs/Success rate. URSL, ureteroscopic lithotripsy. URS $+S$, ureteroscopic lithotripsy with Stone Cone. PCNL, percutaneous nephrolithotomy.

costs of secondary procedures, the average additional cost of URSL with and without an anti-retropulsion device would be $\$ 384$ and \$952, respectively [24]. Our results showed that the group with Stone Cone was not the best cost-effective choice for proximal ureteral calculi in spite of substantially increasing the stone-free rate. The price for labor in health services was very low, but high-tech equipment gave higher margins in China [25]. Goel et al. indicated that PCNL was still a treatment of choice in developing countries for low income [26]. In our cost-analysis, we found that patients without medical insurance preferred PCNL which was invasive and expensive but had higher stone free rate. Higher rate of treatment success would reduce the cost for subsequent therapy, even if the patients would face the higher surgical risk.

This study has limitations. First, it is a single-center study. Second, the sample size is small. Multi-center randomized controlled trials (RCTs) are needed to confirm our results. In conclusion, URSL is an efficacious, safe and cost-effective option for the management of proximal ureteral calculi measuring $\geq 1 \mathrm{~cm}$. Stone Cone increased the treatment success rate but incurred a greater cost burden. Patients without medical insurance would choose PCNL for its higher rate of treatment. Surgeons and patients should consider not only therapeutic efficacy but also the cost impact of each approach.

\section{ACKNOWLEDGEMENTS}

We thank Xuan Zhao for editing the manuscript.

\section{CONFLICT OF INTEREST}

The authors declare that they have no competing interests.

\section{AUTHOR CONTRIBUTIONS}

Jiyu Zhao designed and wrote the main manuscript text, Sheng Yang, Zejun Xiao, Jun Zhang, Jia Liu, Ying Qi help collecting data, Xianen Gu supervised and made the final decision. Jiyu Zhao obtained the funding. All authors reviewed the manuscript.

\section{STATEMENT OF ETHICS}

This study was approved by the Ethical Committee of Beijing Chuiyangliu Hospital and conducted in accordance to the Declaration of Helsinki. All subjects provided written informed consent.

\section{FUNDING SOURCES}

The study was supported by Training Programmed Foundation for the Talents in Beijing (No. 2015000052580G259).

\section{REFERENCES}

[1] Wang Q, Guo J, Hu H, et al. Rigid ureteroscopic lithotripsy versus percutaneous nephrolithotomy for large proximal ureteral stones: A metaanalysis. PLoS One. 2017;12:1-16.

[2] Turk C, Neisius A, Petrik A, et al. Urolithiasis. EAU (European Association of Urology) Guidelines. 2018.

[3] Türk C, Petř́k A, Sarica K, et al. EAU Guidelines on Interventional Treatment for Urolithiasis. Eur Urol. 2016;69:475-482.

[4] Elashry OM, Tawfik AM. Preventing stone retropulsion during intracorporeal lithotripsy. Nat Rev Urol. 2012;9:691-698.

[5] De S, Autorino R, Kim FJ, et al. Percutaneous nephrolithotomy versus retrograde intrarenal surgery: A systematic review and meta-analysis. Eur Urol. 2015;67:125-137.

[6] Nguyen DP, Hnilicka S, Kiss B, et al. Optimization of Extracorporeal 
Shock Wave Lithotripsy Delivery Rates Achieves Excellent Outcomes for Ureteral Stones: Results of a Prospective Randomized Trial. J Urol. 2015;194:418-423.

[7] Lee JW o., Park J, Lee SB a., et al. Mini-percutaneous Nephrolithotomy vs Retrograde Intrarenal Surgery for Renal Stones Larger Than $10 \mathrm{~mm}$ : A Prospective Randomized Controlled Trial. Urology. 2015;86:873-877.

[8] Reis Santos JM. Ureteroscopy from the recent past to the near future. Urolithiasis. 2018;46:31-37.

[9] Wang Y, Zhong B, Yang X, et al. Comparison of the efficacy and safety of URSL, RPLU, and MPCNL for treatment of large upper impacted ureteral stones: A randomized controlled trial. BMC Urol. 2017;17:1-7.

[10] Sozen S, Kupeli B, Tunc L, et al. Management of ureteral stones with pneumatic lithotripsy: report of 500 patients. J Endourol. 2003;17:721724.

[11] Shabana W, Teleb M, Dawod T. Safety and efficacy of using the stone cone and an entrapment and extraction device in ureteroscopic lithotripsy for ureteric stones. Arab J Urol. 2015;13:75-79.

[12] Phan YC, Segaran S, Chew BH, et al. Devices to help combat stone retropulsion during ureteroscopic lithotripsy in 2016. J Clin Urol. 2017; 10:87-92.

[13] Sen H, Bayrak O, Erturhan S, et al. Comparing of different methods for prevention stone migration during ureteroscopic lithotripsy. Urol Int. 2014;92:334-338.

[14] Qi S, Li Y, Liu X, et al. Clinical Efficacy, Safety, and Costs of Percutaneous Occlusive Balloon Catheter-Assisted Ureteroscopic Lithotripsy for Large Impacted Proximal Ureteral Calculi: A Prospective, Randomized Study. J Endourol. 2014;28:1064-1070.

[15] Ceylan K, Sünbül O, Şahin A, et al. Ureteroscopic treatment of ureteral lithiasis with pneumatic lithotripsy: Analysis of 287 procedures in a public hospital. Urol Res. 2005;33:422-425.

[16] Long Q, Guo J, Xu Z, et al. Experience of mini-percutaneous nephrolithotomy in the treatment of large impacted proximal ureteral stones. Urol Int. 2013;90:384-388.
[17] Basiri A, Simforoosh N, Ziaee A, et al. Retrograde, antegrade, and laparoscopic approaches for the management of large, proximal ureteral stones: A randomized clinical trial. Int Braz J Urol. 2009;35:97.

[18] YungShun J, ChingChia L, JungTsung S, et al. Percutaneous nephrostomy for removal of large impacted upper ureteral stones. Kaohsiung J Med Sci. 2007;23:412-416.

[19] Cutler DM. Rising medical costs mean more rough times ahead. JAMA J Am Med Assoc. 2017;318:508-509.

[20] Iacobucci G. UK spent $9.9 \%$ of GDP on healthcare last year, official figures show. BMJ. 2017;357:j2080.

[21] Limb M. A fifth of healthcare spending is wasted, says OECD report. BMJ. 2017;356:j215.

[22] Saleem F, Hassali MA, Iqbal Q, et al. Uncontrollable medicine prices in Pakistan. Lancet. 2016;388:2602.

[23] Wang W, Fan J, Huang G, et al. Prevalence of kidney stones in mainland China: A systematic review. Sci Rep. 2017;7;41630.

[24] Ursiny M, Eisner BH. Cost-effectiveness of anti-retropulsion devices for ureteroscopic lithotripsy. J Urol. 2013;189:1762-1766.

[25] Li H, Dong S, Liu T. Relative efficiency and productivity: A preliminary exploration of public hospitals in Beijing, China. BMC Health Serv Res. 2014; $14 ; 158$.

[26] Goel R, Aron M, Kesarwani PK, et al. Percutaneous antegrade removal of impacted upper-ureteral calculi: still the treatment of choice in developing countries. J Endourol. 2005;19:54-57.

How to cite this article: Jiyu Zhao, Sheng Yang, Zejun Xiao, Jun Zhang, Jia Liu, Ying Qi, Xianen Gu. Comparison of Percutaneous Nephrolithotomy and Ureteroscopic Lithotripsy for Upper Ureteral Calculi. Signa Vitae. 2020;16(2):104-108. doi: $10.22514 /$ sv.2020.16.0054. 